\title{
Desarrollo de plataforma experimental para evaluar el desempeño de controladores PID en un Sistema Térmico
}

\section{Development of experimental platform to evaluate the performance of PID controllers in a Thermal System} CERECERO-NATALE Luis Fidel $\dagger^{*}$, KAUIL-KAUIL Jesús Alejandro, PUC-MAY, José Gerardo y
CHAN-AC, Irvin Arlin

Universidad Tecnológica de la Riviera Maya

ID ${ }^{\text {er }}$ Autor: Fidel, Cerecero-Natale / ORC ID: 0000-0003-3012-3098

ID $1^{\text {er }}$ Coautor: Jesús Alejandro, Kauil-Kauil / ORC ID: 0000-0002-1811-8424

ID $2^{\text {do }}$ Coautor: José Gerardo, Puc-May / ORC ID: 0000-0002-1449-7578

ID $3^{\text {er }}$ Coautor: Irvin Arli, Chan-Ac / ORC ID: 0000-0002-0876-8810

DOI: $10.35429 /$ JEA.2019.19.6.11.16

Recibido: 19 de Abril 2019; Aceptado 30 Junio, 2019

\begin{abstract}
Resumen
En este artículo se presenta el desarrollo de la programación y la sintonización de las ganancias de control para un sistema térmico, la programación se realizó en el software LabVIEW donde es posible visualizar y manipular los sensores, indicadores y actuadores, los datos se encuentran representados en una interfaz gráfica de usuario. El bloque Digital Write se utiliza en el LED RGB para determinar el nivel de temperatura con identificación de colores, también se utilizó el bloque Analog Read para leer los datos del sensor de temperatura LM35, la adquisición de datos se obtuvo por medio del datalogger, en donde se estructuran los datos generados del experimento en un bloc de notas en la PC, la comunicación se realiza a través de MakerHub en donde se selecciona el tipo de conexión y el puerto para enviar datos de LabVIEW a la tarjeta Arduino, para determinar la curva tangencial con los parámetros del experimento, con el objetivo de aplicar el primer método de Ziegler-Nichols, adquiriendo las constantes $K_{p}, T_{i}$ y $T_{d}$ del sistema cerrado, para evaluar los controladores P, PI y PID. Para sintonizar las ganancias del Controlador Proporcional-IntegralDerivativo (PID) se utiliza el software Excel, donde se aplican las fórmulas del primer método de Ziegler-Nichols, una vez que se ajustan las ganancias, es posible realizar experimentos en tiempo real. Con los archivos almacenados por medio del sistema de adquisición de datos (DAQ), se puede evaluar el desempeño de los controladores con diferentes ganancias, es decir comparar los datos de entrada y de salida.
\end{abstract}

Controlador PID, LabVIEW, Arduino

\begin{abstract}
This article presents the development of the programming and the tuning of the control gains for a thermal system, the programming was carried out in the LabVIEW software where it is possible to visualize and manipulate the sensors, indicators and actuators, the data is represented in a graphical user interface. The Digital Write block is used in the RGB LED to determine the temperature level with color identification, the Analog Read block was also used to read the data of the temperature sensor LM35, the data acquisition was obtained through the datalogger, where they are stored and save the data generated from the experiment in a notepad on the PC, the communication is done through MakerHub where the type of connection and the port to send LabVIEW data to the Arduino card is selected, to determine the tangential curve with the parameters of the experiment, with the objective of applying the first Ziegler-Nichols method, acquiring the $K_{p}, T_{i}$ y $T_{d}$ gains of the closed system, to evaluate the P, PI and PID controllers. To tune the gains of the Proportional-Integral-Derivative Controller (PID) the Excel software is used, where the formulas of the first Ziegler-Nichols method are applied, once the gains are adjusted, it is possible to do real-time experiments. With the files stored through the data acquisition system (DAQ), you can evaluate the performance of the controllers with different gains, that is, compare the input and output data.
\end{abstract}

PID controller, LabVIEW, Arduino

Citación: CERECERO-NATALE Luis Fidel, KAUIL-KAUIL Jesús Alejandro, PUC-MAY, José Gerardo y CHAN-AC, Irvin Arlin. Desarrollo de plataforma experimental para evaluar el desempeño de controladores PID en un Sistema Térmico. Revista de Aplicaciones de la Ingeniería. 2019. 6-19: 11-16

\footnotetext{
* Correspondencia del Autor (Correo electrónico: mecatronica.cerecero@gmail.com)

$\dagger$ Investigador contribuyendo como primer autor.
} 


\section{Introducción}

La ingeniería de control es una disciplina que se enfoca en modelar matemáticamente una gama diversa de sistemas dinámicos, el diseño de controladores que harán que estos sistemas se comporten en la manera deseada. Dispositivos como circuitos eléctricos, procesadores digitales y los microcontroladores son muy utilizados en todo sistema de control moderno. La ingeniería de control tiene un amplio rango de aplicación en áreas como los sistemas de vuelo, de propulsión de los aviones de aerolíneas, militares, en la carrera espacial y últimamente en la industria automotriz.

El acrónimo PID proviene de las tres partes que lo componen: una acción Proporcional, una acción Integral y una acción Derivativa. El control PID es un sistema de regulación que aprovecha las ventajas de cada uno de los controladores de acciones básicas, de manera que, si la señal de error varía lentamente en el tiempo, predomina la acción proporcional e integral mientras que, si la señal de error varía rápidamente, predomina la acción derivativa. Tiene la ventaja de ofrecer una respuesta muy rápida y una compensación de la señal de error inmediata en el caso de perturbaciones (Zhao, 1993).

La sintonización de controladores (Ogata, 2010) se refiere a la selección de aquellos valores, de las constantes $K_{p}, T_{i}$, y $T_{d}$, para obtener el comportamiento deseado del controlador. Ziegler y Nichols proponen reglas para determinar valores de la ganancia proporcional $K_{p}$, Tiempo integral $T_{i}$ y Tiempo derivativo $T_{d}$ basados en las características de la respuesta transitoria de una planta dada. Existen dos métodos llamados reglas de sintonización de Ziegler-Nichols.

En el primer método se obtiene de manera experimental la respuesta de una planta ante la entrada de un escalón unitario; si la planta no tiene integradores ni polos complejos conjugados, la curva de respuesta ante el escalón unitario se debe de ver, con forma de $S$. Este método se aplica si la respuesta a una entrada escalón presenta una forma de $S$. Tal respuesta puede ser generada experimentalmente una simulación de la dinámica de la planta.
La curva $S$ puede ser caracterizada por dos constantes, Tiempo de retraso $L$ y constante de tiempo $T$; estas constantes son determinadas por el método gráfico, donde se traza una línea tangente en el punto de inflexión de la curva $S$ y se determinan las intersecciones de la línea tangente con la línea del eje del tiempo $c(t)=K$, tal como se muestra en la Figura 1.

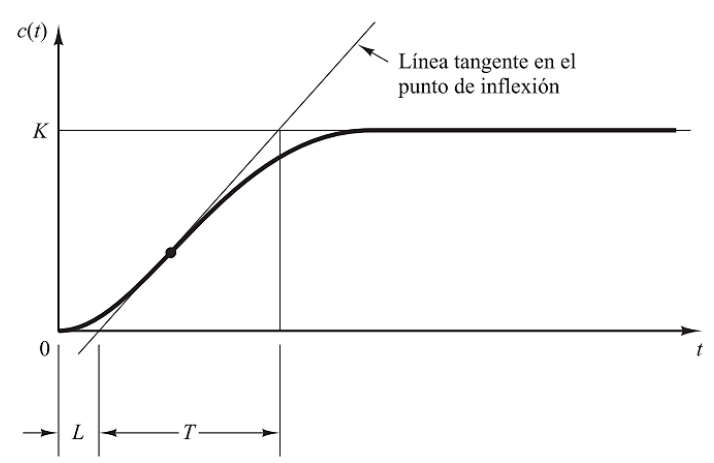

Figura 1 Curva de respuesta en forma de $S$

La función de transferencia representa la respuesta del sistema dinámico que relaciona la salida sobre la entrada, puede ser aproximada a un modelo de primer orden con retardo tal como se muestra en la ecuación (1).

$\frac{\operatorname{Salida}(s)}{\operatorname{Entrada}(s)}=\frac{K}{T s+1} e^{-L s}$

\section{Desarrollo de la interfaz de control gráfica}

El desarrollo de la interfaz gráfica se realiza para la plataforma experimental mostrada en (Cerecero, Puc, Ceme, Kauil, \& Chan, 2019). El LINX LabVIEW MakerHub facilita la conexión con plataformas embebidas comunes como chipKIT, Arduino y NI myRIO, así como sensores comunes incluyendo acelerómetros, sensores de temperatura y sensores ultrasónicos de distancia.

En este firmware permite comunicar el Arduino con LabVIEW a partir de opciones seleccionables desde la barra de herramientas del programa, sin la necesidad de cargar programa alguno al Arduino de manera independiente (Ramses, 2017). 


\section{Procedimiento de enlace:}

1. Se descarga el complemento de MakerHub en la página oficial de National Instruments (MakerHub, 2019). Una vez instalada, aparece en el panel de herramientas de LabVIEW, en la barra desplegable se selecciona la opción de MakerHub. Se abre una ventana emergente donde se configura el tipo y puerto serial del Arduino UNO, para cargar el enlace y finalizar.

2. Ya cargado el programa se ejecuta y se visualizan los parámetros de los sensores y actuadores de la interfaz de LabVIEW.

En la Figura 2 se muestra un diagrama a bloques de cómo se realiza el enlace entre LabVIEW y Arduino utilizando la librería de MakerHub.

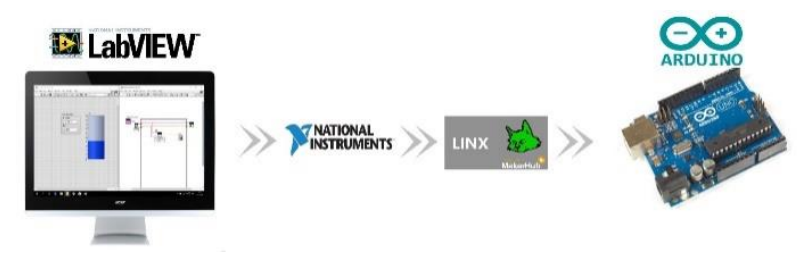

Figura 2 Diagrama a bloques del enlace entre LabVIEW y Arduino

En la Figura 3 se muestra la Interfaz Gráfica de Usuario (GUI) de la plataforma, donde se realiza la programación de un algoritmo en lenguaje gráfico utilizando la librería de MakerHub para utilizar Arduino UNO como tarjeta de Adquisición de Datos (DAQ) con lo que se pueden leer las entradas analógicas, escribir salidas reguladas con Modulación por Ancho de Pulso (PWM) y salidas digitales (Zambrano \& Villamar Bravo, 2015).

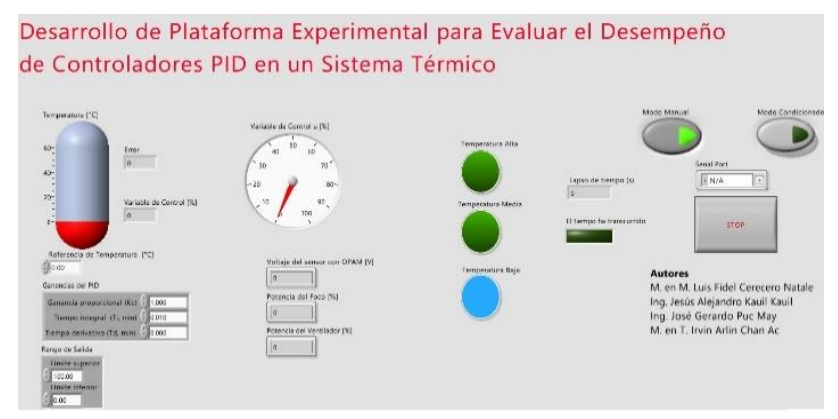

Figura 3 Interfaz de la plataforma experimental en LabVIEW
La interfaz LINX permite la conexión entre el software Arduino y LabVIEW, el bloque control de INIT que permite seleccionar el puerto COM al cual está conectado el Arduino para iniciar la comunicación desde la PC, controles del bloque INIT en los que se configura el tipo de conexión y el tipo de tarjeta Arduino que se utiliza.

La VISA es un API de alto nivel utilizado para comunicarse con buses de instrumentación, es independiente de la plataforma del bus y del entorno. El USB es un bus de comunicación basado en mensajes. Esto significa que una PC y un dispositivo USB se comunican enviando comandos y datos a través del bus en forma de texto o datos binarios.

En el software LabVIEW se utiliza un bucle While Loop, se usaron bloques de señales digitales en donde las señales toman valores de 0 y 1 , con las que son activadas las señales digitales del LED RGB que responden de acuerdo al rango de temperatura al que se encuentra sometido el sistema cerrado, es decir:

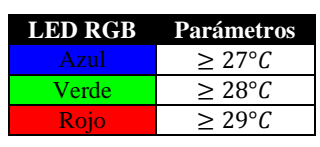

Indicando el nivel de temperatura, las lecturas de las señales analógicas tienen un rango de $0 \mathrm{~V}$ a $5 \mathrm{~V}$ con una variación acorde al tiempo, la alimentación de la instrumentación es de $12 \mathrm{~V}$ y se multiplica por una constante para calibrar y adquirir la temperatura en grados Celsius del sistema térmico, las señales PWM tiene un rango de porcentaje que varía el 100\% de su capacidad, regulando la salida de voltaje de $12 \mathrm{~V}$ en corriente directa de los actuadores (ventilador y foco).

La adquisición de datos se obtiene a partir de un datalogger, en donde se estructuran y guardan dichos datos en un bloc de notas de la PC (José Rafael Lajara Vizcaíno, 2011), se almacenan los parámetros del tiempo, la temperatura, porcentajes de encendido del foco y el ventilador. En Tabla 1 se muestra el nombre del Instrumento Virtual (VI) y una breve descripción del funcionamiento de los bloques utilizados en el desarrollo de la plataforma experimental para evaluar el desempeño de los controladores PID en el software LabVIEW, por otra parte, en la Figura 4 se ilustra el Diagrama a Bloques de la programación dividido en cinco secciones. 
En la Sección 1 es posible configurar el tiempo total en el que se realizan los experimentos. En la Sección 2 se asignan los pines de entrada y salida de la tarjeta en este caso se utilizan entradas analógicas, salidas digitales y salidas Moduladas en Anchos de Pulso (PWM). En la Sección 3 se pueden configurar los límites de temperatura para indicar el encendido del LED RGB. En la Sección 4 se ajustan las ganancias del controlador PID y los límites de los rangos de operación de las señales que controlan los actuadores, cabe mencionar que los valores pueden ser modificados desde el Panel Frontal.

En la Sección 5 se muestra la estructura de adquisición de datos tipo datalogger, los datos registrados durante los experimentos son estructurados y almacenados en un bloc de notas con el que es posible realizar el análisis y la graficación de los resultados fuera de línea utilizando programas como Excel o MATLAB

\begin{tabular}{|c|c|}
\hline Bucle & Puerto serial \\
\hline$\square$ & 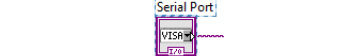 \\
\hline $\begin{array}{l}\text { Repite el código dentro de su } \\
\text { subdiagrama hasta que se produce una } \\
\text { condición específica. Un bucle siempre } \\
\text { se ejecuta al menos una vez. }\end{array}$ & $\begin{array}{l}\text { Abre una conexión serie a un } \\
\text { dispositivo LINX remoto. }\end{array}$ \\
\hline Serie abierta & Construir matriz \\
\hline 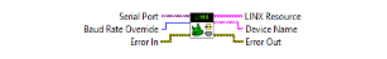 & 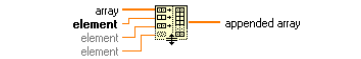 \\
\hline $\begin{array}{l}\text { Abre una conexión serie a un } \\
\text { dispositivo LINX remoto. }\end{array}$ & $\begin{array}{l}\text { Concatena múltiples matrices o } \\
\text { agrega elementos a una matriz de } n \\
\text { dimensiones. }\end{array}$ \\
\hline Booleano & Escritura digital \\
\hline DBE & 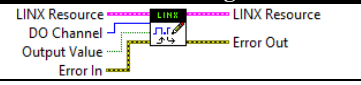 \\
\hline $\begin{array}{l}\text { Número puede ser un número escalar, } \\
\text { una matriz o un grupo de números, una } \\
\text { matriz de grupos de números, etc. }\end{array}$ & $\begin{array}{l}\text { Escribe los valores en los canales de } \\
\text { salida digital especificados. }\end{array}$ \\
\hline Construir matriz & Nodo de retroalimentación \\
\hline 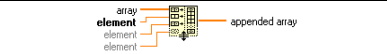 & 获 \\
\hline $\begin{array}{l}\text { Concatena múltiples matrices o agrega } \\
\text { elementos a una matriz de } n \\
\text { dimensiones. }\end{array}$ & $\begin{array}{l}\text { Almacena datos de una ejecución de } \\
\text { VI o iteración de bucle a la } \\
\text { siguiente. }\end{array}$ \\
\hline Lectura analógica de 1 canal & Funciones booleanas \\
\hline 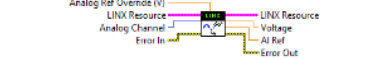 & 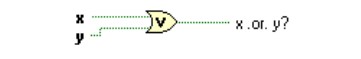 \\
\hline $\begin{array}{l}\text { Lee el valor del canal de entrada } \\
\text { analógico especificado. }\end{array}$ & $\begin{array}{l}\text { Calcula el OR lógico de las } \\
\text { entradas. Ambas entradas deben ser } \\
\text { valores booleanos, valores } \\
\text { numéricos o grupos de errores. Si } \\
\text { ambas entradas son FALSO, la } \\
\text { función devuelve FALSO. De lo } \\
\text { contrario, devuelve VERDADERO. }\end{array}$ \\
\hline $\begin{array}{l}\text { Funciones de comparación (menos o } \\
\text { igual) }\end{array}$ & Verdadero o Falso \\
\hline$x-\quad \geqslant \quad x>y ?$ & TF \\
\hline $\begin{array}{l}\text { Devuelve VERDADERO si } x \text { es } \\
\text { mayor o igual que } y \text {. De lo contrario, } \\
\text { esta función devuelve FALSO. Puede } \\
\text { cambiar el modo de comparación de } \\
\text { esta función. Puede comparar una } \\
\text { matriz o agrupación de un tipo de datos } \\
\text { con un escalar del mismo tipo de datos } \\
\text { y generar una matriz o agrupación de } \\
\text { valores booleanos. El panel del } \\
\text { conector muestra los tipos de datos } \\
\text { predeterminados para esta función } \\
\text { polimórfica. }\end{array}$ & $\begin{array}{l}\text { El uso del punto decimal del } \\
\text { sistema define el separador } \\
\text { decimal. Si es VERDADERO } \\
\text { (predeterminado), el separador } \\
\text { decimal usa el separador decimal } \\
\text { localizado. Si es FALSO, el } \\
\text { separador decimal es un punto. }\end{array}$ \\
\hline
\end{tabular}

\begin{tabular}{|c|c|}
\hline Booleano a $(0,1)$ & $\begin{array}{l}\text { Funciones de comparación } \\
\text { (multiplicador) }\end{array}$ \\
\hline Boolean p p1:0- & $x>x^{x} y$ \\
\hline $\begin{array}{l}\text { Convierte un valor FALSO o } \\
\text { VERDADERO booleano en un entero } \\
\text { de } 16 \text { bits con un valor de } 0 \text { o } 1 \text {, } \\
\text { respectivamente. }\end{array}$ & $\begin{array}{l}\text { Devuelve el producto de las } \\
\text { entradas. Si conecta dos valores de } \\
\text { forma de onda o dos valores de tipo } \\
\text { de datos dinámicos a esta función, } \\
\text { aparecerán terminales de error in y } \\
\text { error out en la función. El panel del } \\
\text { conector muestra los tipos de datos } \\
\text { predeterminados para esta función } \\
\text { polimórfica. }\end{array}$ \\
\hline PID Vis & Ganancias PID \\
\hline 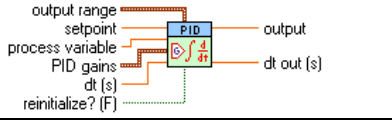 & 奇骂 \\
\hline $\begin{array}{l}\text { El algoritmo PID presenta un límite de } \\
\text { rango de salida de control con salida de } \\
\text { controlador anti-cuerda y sin } \\
\text { perturbaciones del integrador para } \\
\text { cambios de ganancia PID. Utiliza la } \\
\text { instancia DBL de este VI para } \\
\text { implementar un solo bucle de control. } \\
\text { Utilice la instancia DBL Array para } \\
\text { implementar el control paralelo de } \\
\text { múltiples bucles. }\end{array}$ & $\begin{array}{l}\text { Las ganancias PID especifican la } \\
\text { ganancia proporcional, el tiempo } \\
\text { integral y el parámetro de tiempo } \\
\text { derivativo del controlador. }\end{array}$ \\
\hline Numera la cadena fraccionaria & $\begin{array}{l}\text { Funciones de comparación } \\
\text { (seleccionador) }\end{array}$ \\
\hline 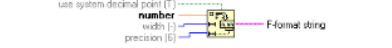 & $\gg$ s?th \\
\hline $\begin{array}{l}\text { Convierte el número a un formato } F \\
\text { (notación fraccional), cadena de punto } \\
\text { flotante al menos ancho de caracteres } \\
\text { de ancho o más ancho si es necesario. } \\
\text { El panel del conector muestra los tipos } \\
\text { de datos predeterminados para esta } \\
\text { función polimórfica. }\end{array}$ & $\begin{array}{l}\text { Devuelve el valor cableado a la } \\
\text { entrada } t \text { o entrada } f, \text { dependiendo } \\
\text { del valor de } s . \mathrm{Si} s \text { es } \\
\text { VERDADERO, esta función } \\
\text { devuelve el valor cableado a } t \text {. Si s } \\
\text { es FALSO, esta función devuelve el } \\
\text { valor cableado a } f \text {. }\end{array}$ \\
\hline Cuerdas de concatenación & $\begin{array}{l}\text { Establece el ciclo de trabajo en e1 } \\
\text { canal }\end{array}$ \\
\hline 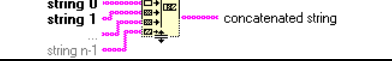 & 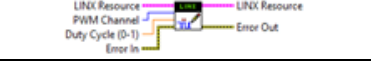 \\
\hline $\begin{array}{l}\text { Concatena cadenas de entrada y } \\
\text { matrices 1D de cadenas en una sola } \\
\text { cadena de salida. Para las entradas de } \\
\text { matriz, esta función concatena cada } \\
\text { elemento de la matriz. }\end{array}$ & $\begin{array}{l}\text { Establece el ciclo de trabajo del } \\
\text { canal PWM especificado. Un ciclo } \\
\text { de trabajo de } 0 \text { corresponde al } 0 \% \\
\text { activado y un ciclo de trabajo de } 1 \\
\text { corresponde al } 100 \% \text { activado. }\end{array}$ \\
\hline $\begin{array}{l}\text { Escritura hoja de cálculo delimitada } \\
\text { VI }\end{array}$ & Cierre \\
\hline 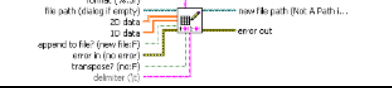 & 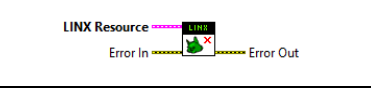 \\
\hline $\begin{array}{l}\text { Convierte una matriz 2D o 1D de } \\
\text { cadenas, enteros con signo o números } \\
\text { de doble precisión en una cadena de } \\
\text { texto y escribe la cadena en un nuevo } \\
\text { archivo de flujo de bytes o agrega la } \\
\text { cadena a un archivo existente. este VI } \\
\text { para transponer o separar datos. }\end{array}$ & $\begin{array}{l}\text { Cierre la conexión con el dispositivo } \\
\text { LINX remoto y libere todos los } \\
\text { recursos de } \mathrm{E} \text { / S locales. }\end{array}$ \\
\hline Funciones de comparación (Divisor) & $\begin{array}{l}\text { Componentes de una estructura } \\
\text { de caso }\end{array}$ \\
\hline 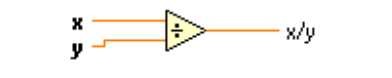 & 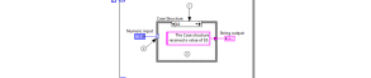 \\
\hline $\begin{array}{l}\text { Calcula el cociente de las entradas. } \mathrm{Si} \\
\text { conecta dos valores de forma de onda } \\
\text { o dos valores de tipo de datos } \\
\text { dinámicos a esta función, aparecerán } \\
\text { terminales de error (entrada) y error } \\
\text { (salida) en la función. El panel del } \\
\text { conector muestra los tipos de datos } \\
\text { predeterminados para esta función } \\
\text { polimórfica. }\end{array}$ & $\begin{array}{l}\text { Contiene uno o más subdiagramas, } \\
\text { o casos, exactamente uno de los } \\
\text { cuales se ejecuta cuando se ejecuta } \\
\text { la estructura. El valor conectado al } \\
\text { selector de casos determina qué caso } \\
\text { ejecutar. }\end{array}$ \\
\hline Tiempo transcurrido & $\begin{array}{l}\text { Funciones de comparación } \\
\text { (seleccionador) }\end{array}$ \\
\hline 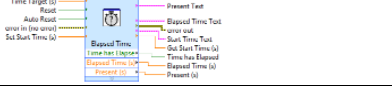 & $\begin{array}{l}t \rightarrow \infty \\
t-\infty\end{array}$ \\
\hline $\begin{array}{l}\text { Indica la cantidad de tiempo que ha } \\
\text { transcurrido desde la hora de inicio } \\
\text { especificada. }\end{array}$ & $\begin{array}{l}\text { Devuelve el valor cableado a la } \\
\text { entrada } t \text { o entrada } f \text {, dependiendo } \\
\text { del valor de } s . \mathrm{Si} s \text { es } \\
\text { VERDADERO, esta función } \\
\text { devuelve el valor cableado a } t \text {. Si s } \\
\text { es FALSO, esta función devuelve el } \\
\text { valor cableado a } f \text {. }\end{array}$ \\
\hline
\end{tabular}

Tabla 1 Icono y descripción de los componentes utilizados en el diagrama de bloque de LabVIEW 


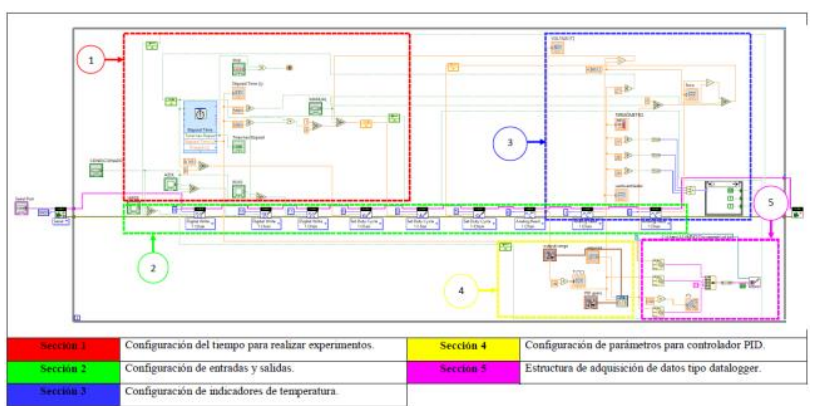

Figura 4 Diagrama a Bloques de la programación en LabVIEW dividido en 5 secciones

\section{Sintonización de ganancias del controlador PID}

El Controlador Proporcional, Integral y Derivativo reúne las ventajas de cada una de las tres acciones de control individuales. $\mathrm{La}$ ecuación de un controlador PID con esta acción combinada se obtiene con la ecuación (2).

$u(t)=K_{p} e(t)+\frac{K_{p}}{T_{i}} \int_{0}^{t} e(\tau) d \tau+K_{p} T_{d} \frac{d e(t)}{d t}$

\section{Experimentación}

El experimento se realiza ejecutando el programa en modo manual, la secuencia comienza cuando la lectura de la temperatura inicial es de $25^{\circ} \mathrm{C}$, la prueba experimental dura un lapso de $30 s$, en donde los primeros $20 s$ el actuador (foco) permanece encendido a su máxima intensidad expresada en porcentaje y los $10 s$ restantes se apaga, cabe mencionar que el potenciómetro sirve como divisor de voltaje, en donde la variación de su posición regula de $0 \%$ a $100 \%$ de la capacidad del actuador, a partir de ello, se adquieren los datos por medio de LabVIEW y se registran por medio del datalogger, una vez obtenidos los datos con los parámetros del tiempo, temperatura y porcentaje de los actuadores, los datos se presentan en una gráfica de tipo dispersión a través del software Microsoft Office Excel.

En el Gráfico 1 se muestra la respuesta al escalón para aplicar el primer método de Ziegler-Nichols, con lo que se realiza la identificación paramétrica empleando el método gráfico, en el cual se traza una línea tangencial en el punto de inflexión en el eje $x$, en donde se encuentra el tiempo y en el eje $y$ la temperatura, para determinar el valor del tiempo de retardo $L$, el valor de la ganancia del sistema $K$ (se obtiene al calcular la diferencia de la temperatura final y la temperatura inicial).

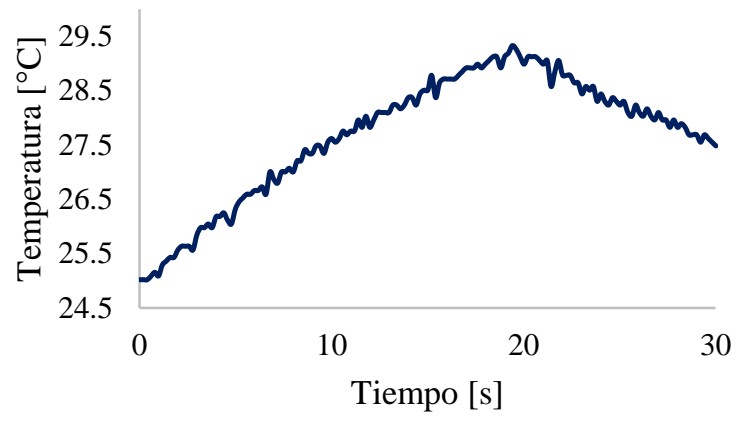

Gráfico 1 Respuesta al escalón

Los parámetros de $K, L$ y $T$ expresados en la ecuación (1) han sido identificados y se presentan en la ecuación (3) que modela el comportamiento matemático en una función de transferencia de primer orden con retardo (Ziegler, 1942).

$$
\frac{\operatorname{Salida}(s)}{\operatorname{Entrada}(s)}=\frac{4.31}{12.24+1} e^{-0.6 s}
$$

Una vez definidos los parámetros $K, L \mathrm{y}$ $T$, el software Microsoft Office Excel se encarga de realizar las operaciones en donde se ingresaron las fórmulas de sintonización del primer método de Ziegler-Nichols, los resultados de $K_{p}, T_{i}$ y $T_{d}$ están dados en segundos, pero el Tiempo integral $\left(T_{i}\right)$ y el Tiempo derivativo $\left(T_{d}\right)$ de las constantes del PID que se utilizan en el software LabVIEW trabajan con valores en minutos como se muestra en la Tabla 2, por lo cual se emplea el sistema sexagesimal para realizar la conversión de segundos a minutos.

\begin{tabular}{|l|r|r|r|}
\hline Controlador & \multicolumn{1}{c}{$\boldsymbol{K}_{\boldsymbol{p}}$} & \multicolumn{1}{c|}{$\boldsymbol{T}_{\boldsymbol{i}}$} & \multicolumn{1}{c|}{$\boldsymbol{T}_{\boldsymbol{d}}$} \\
\hline $\mathbf{P}$ & 20.40 & $\infty$ & 0 \\
\hline PI & 18.36 & 0.0333333 & 0 \\
\hline PID & 24.48 & 0.023 & 0.005 \\
\hline
\end{tabular}

Tabla 2 Regla de sintonización del primer método de Ziegler-Nichols en minutos

En la programación se cambia la opción de modo manual a modo condicionado para introducir las ganancia $K_{p}$, las constantes de tiempo $T_{i}$ y $T_{d}$ en minutos para cada señal P, PI y PID. Se establece un límite de tiempo de 200 segundos para el experimento y se activa la opción restablecer automáticamente después que se cumpla el lapso de tiempo asignado (Instruments, 2012). 


\section{Comparación de los controladores P, PI y PID}

Para evaluar el desempeño del controlador PID y sus variantes se utiliza la estructura presentada en la Tabla 3 donde los datos son proporcionados por el datalogger. Se puede apreciar que la mejor opción para este sistema térmico, sin perturbación, es el controlador $\mathrm{P}$, ya que su comportamiento y tiempo de respuesta es eficaz al alcanzar la referencia asignada.

\begin{tabular}{|c|c|c|c|c|}
\hline $\begin{array}{l}\text { Tiempo } \\
{[\mathrm{s}]}\end{array}$ & $\begin{array}{c}\text { Control P } \\
{\left[{ }^{\circ} \mathbf{C}\right]}\end{array}$ & $\begin{array}{c}\text { Control PI } \\
{\left[{ }^{\circ} \mathrm{C}\right]}\end{array}$ & $\begin{array}{c}\text { Control PID } \\
{\left[{ }^{\circ} \mathbf{C}\right]}\end{array}$ & $\begin{array}{c}\text { Referencia } \\
{\left[{ }^{\circ} \mathbf{C}\right]}\end{array}$ \\
\hline 0 & 25.01953 & 25.22087 & 25.08789 & 30 \\
\hline 0.79214 & 25.22460 & 25.08789 & 25.1562 & 30 \\
\hline 0.38126 & 25.15625 & 25.01953 & 25.08789 & 30 \\
\hline 0.56940 & 25.08789 & 25.01953 & 25.15625 & 30 \\
\hline & & & & \\
\hline 199.8862 & 29.941406 & 29.94140 & 29.94140 & 30 \\
\hline
\end{tabular}

Tabla 3 Comparativo de controladores para regular temperatura (estructura del datalogger)

Para evaluar el desempeño del sistema en lazo cerrado se calcula la integral del valor absoluto del error (IAE) (Roger Moliner, 2016), expresada mediante la ecuación (4).

$I A E=\int_{0}^{T}|e(t)| d t$

Una forma de conocer la energía consumida durante los experimentos es calculando la integral del valor absoluto de la entrada de control (E) (Sánchez, 2006), como se muestra en la ecuación (5):

$E=\int_{0}^{t}|U(\tau)| d \tau$

En la Tabla 4 se muestra la comparación de la respuesta de los controladores para el caso de regulación de posición, la última columna (S/s) representa la cantidad de muestras por segundo de cada controlador.

\begin{tabular}{|l|r|c|c|}
\hline Controlador & \multicolumn{1}{c}{ IAE } & \multicolumn{1}{c|}{ E } & S/s \\
\hline P & $\mathbf{3 7 6 . 4 9 4 2 5}$ & $\mathbf{7 6 7 8 . 4 6 4 7 7}$ & $\mathbf{7}$ \\
\hline PI & 392.11924 & 16801.4429 & 6 \\
\hline PID & 382.50986 & 21831.3483 & 6 \\
\hline
\end{tabular}

Tabla 4 Comparativo de los controladores para el caso de regularización

En el Gráfico 2 se logra apreciar la comparación de la regulación de temperatura en grados centígrados del sistema, donde se observan las respuestas en función al tipo de control que se está utilizando al asignar una referencia fija.
Por otra parte, en el Gráfico 3, podemos observar la variable de control para llegar a la referencia de temperatura de cada uno de los controladores, donde la potencia del actuador está expresada en porcentaje, en la Gráfico 4, se ilustra la señal error al regular la temperatura.

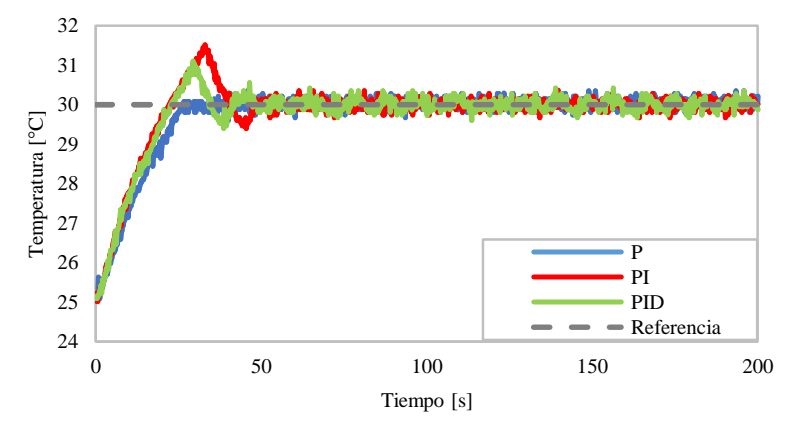

Gráfico 2 Comparativo de controladores P, PI y PID (respuesta del sistema)

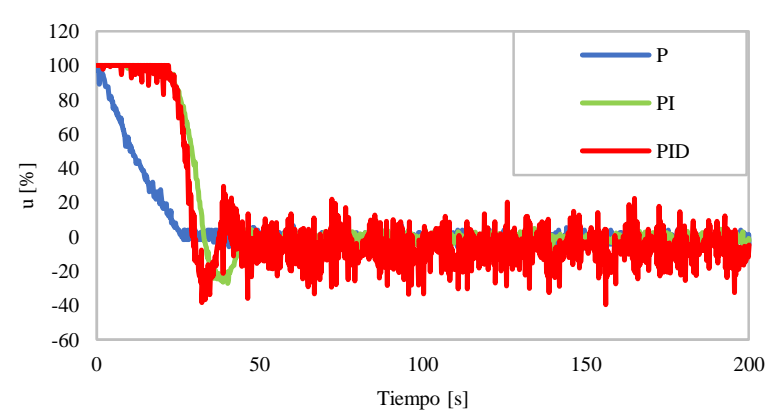

Gráfico 3 Comparativo de controladores P, PI y PID (variable de control)

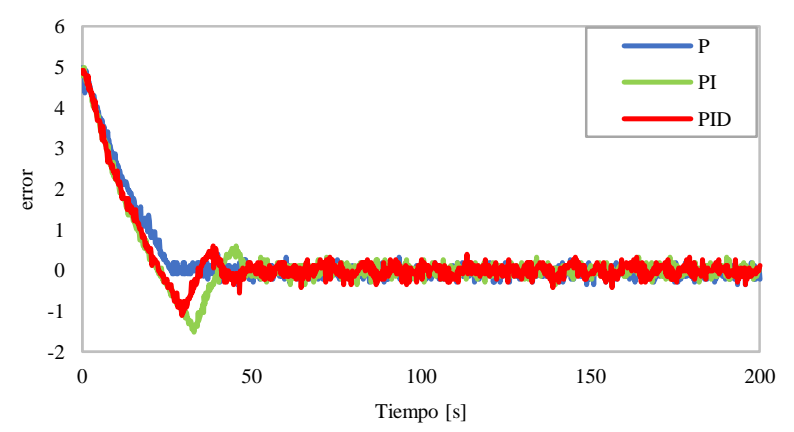

Gráfico 4 Comparativo de los controladores P, PI y PID (Error)

\section{Agradecimiento}

Le agradecemos a la Universidad Tecnológica de la Riviera Maya, en especial a la Dirección Académica y a la Subdirección de la Ingeniería en Mantenimiento Industrial por el apoyo brindado para el registro de este artículo.

\section{Conclusiones}

Se logró diseñar, construir, instrumentar y controlar un sistema térmico para realizar pruebas de control, donde se puede contrastar el desempeño del controlador PID y sus diversas configuraciones de manera satisfactoria. 
Una vez integrada la plataforma experimental con los elementos mecánicos y electrónicos se procedió a desarrollar una interfaz gráfica para el control de los experimentos en un sistema térmico de lazo cerrado, donde se lograron calcular las ganancias del controlador PID usando el primer método de Ziegler-Nichols en una hoja de cálculo en el software Excel, con dichos resultados se procedió a sintonizar el control en la interface de LabVIEW de National Instruments utilizando la librería de MakerHub como enlace de comunicación con el Arduino UNO, dicho sistema permite capturar los datos para procesar fuera de línea en un archivo tipo datalogger de manera eficaz. Con los datos obtenidos en forma de tablas es posible graficar la respuesta de los sistemas con distintos valores de ganancias para evaluar el desempeño de los controladores.

Como trabajo futuro, se pretende evaluar el desempeño de los controladores PID sometidos a perturbaciones externas, ya que hasta el momento, las pruebas han sido realizadas en lazo cerrado sin perturbación, cabe mencionar que la tarjeta electrónica tipo shield cuenta con otro potenciómetro para regular la velocidad del segundo actuador (ventilador), con lo que es posible inducir un cambio repentino de la temperatura en el recipiente, lo cual es muy interesante para visualizar, comparar y evaluar el desempeño de las acciones de control integral y derivativa, ya que, hasta el momento en las pruebas sin perturbación el controlador mejor evaluado es el proporcional.

Con esta plataforma experimental los estudiantes de nivel licenciatura pueden aprender diversas disciplinas de la ingeniería con el enfoque de aprendizaje basado en problemas $\mathrm{y}$ en proyectos, donde desarrollan habilidades prácticas que están fundamentadas en teoría. Es una herramienta para facilitar el aprendizaje de los sistemas de control y otras áreas como: la electrónica analógica, electrónica digital, electrónica de potencia, programación de microcontroladores, automatización, interfaces gráficas, metrología y diseño industrial utilizando las ciencias básicas, lo que contribuye de manera sustancial al aprendizaje de los estudiantes.

\section{Referencias}

Blanes, C. S. (2011). Diseño mecánico con Autodesk Inventor paso a paso. San Vicente (Alicante): Editorial Club Universitario.

Cerecero, L. F., Puc, J., Ceme, J., Kauil, J., \& Chan, I. (2019). Desarrollo de Plataforma Experimental para Sistemas Térmicos Empleando Métodos de Sintonización. Academia Jornals, Volúmen 11, (4), ISSN 19465351, 1-9.

José Rafael Lajara Vizcaíno, J. P. (2011). LabVIEW: Entorno gráfico de programación. Barcelona: Marcombo.

MakerHub. (20 de 01 de 2019). LabVIEW MakerHub. Obtenido de https://www.labviewmakerhub.com/

Medina, C. (s.f.). Desarrollo de Módulos Gráficos para la Enseñanza-Aprendizaje en los Cursos de Comunicaciones. I+D Tecnológico, 8(2), 24-29 ISSN: 1680-8894, https://revistas.utp.ac.pa/index.php/idtecnologico/article/view/91.

National Instuments. (2010). LabVIEW TM Core 1 Manual de Curso.

Ogata, K. (2010). Ingeniería de control moderna. Madrid: Pearson Educación.

Ramses, C. C. (2017). Diseño de un módulo electrónico e interfaz para el control de un posicionado 3D. Tesis de ingeniería, Universidad Nacional Autónoma de México, Departamento de Ingeniería en Sistemas Computacionales y Automatización, Cuidad Universitaria.

Roger Moliner, R. T. (2016). Herramienta para la sintonía robusta de controladores PI/PID de dos grados de libertad. ScienceDirect, 10.

Sánchez, J. A. (2006). Instrumentación y control avanzado de procesos. España: Díaz de Santos.

Ziegler, J. y. (1942). Optimus aetings for automatic controls. EUA: ASME Transactions. 